\title{
Evaluation of Porphyromonas Gingivalis in Periodontitis Patients of Met.S and Periodontitis Patients without Met.S
}

\author{
Dr. Ashwini Walke ${ }^{1}$, Dr. Tushar Bhagat ${ }^{2}$, Dr. Abdulmajeed Mutairan Alanazi ${ }^{3}$ \\ ${ }^{1}$ MDS, Department of oral pathology and microbiology, Riyadh, Saudi Arabia. \\ ${ }^{2}$ MDS, Department of prosthodontics, Prince Sattam bin abdulaziz university Riyadh, Saudi Arabia. \\ ${ }^{3}$ BDS (Intern), Prince Sattam bin abdulaziz university Riyadh, Saudi Arabia. \\ ashwiniwalke2210@gmail.com
}

*Corresponding Author: Dr. Ashwini Walke, Department of oral pathology and microbiology, Saudi Arabia.

\begin{abstract}
Oral cavity is said to be the mirror of systemic health. Many systemic diseases first manifest in the oral cavity. Also an oral disease has a potential to act as an independent risk factor for causing systemic disease. This bidirectional view is gaining acceptance currently, due to findings that, association between periodontal disease and systemic conditions such as Metabolic Syndrome. Effort has brought advances in revealing the etiological and pathological links between the chronic inflammatory dental disease, periodontitis and systemic condition, Metabolic Syndrome. The role of microorganisms in periodontal disease is well documented. In the last 10 years, studies have been published indicating a positive or negative relationship between periodontitis and various systemic diseases, including Met.S. The most frequently isolated microaerophilic pathogens are (A.actinomyctemcomitans, Campylobacter rectus, and Eikenellacorrodens) and anaerobic pathogens are (P.gingivalis, Bacteroidesforsythus, T.denticola, Prevotella intermedia, Fusobacterium nucleatum, Eubacterium, and spirochetes). In the present study P.gingivalis periodontal pathogen confirmed increased colonization of periodontal pathogens, are cultured in both periodontitis patients without Met.S and Periodontitis patients with Met.S.
\end{abstract}

Keywords: Metabolic Syndrome, Periodontitis, Porphyromonas gingivalis

\section{INTRODUCTION}

According to WHO Report 2008, In India, 53\% of the deaths occurs due to Non Communicable Diseases (NCD). These NCDs are the result of lifestyle related behavioral risk factors such as use of tobacco, physical inactivity, unhealthy diet and harmful use of alcohol leading to metabolic/physiological changes like increased in blood pressure (BP), overweight/obesity, raised blood glucose and raised cholesterol levels. ${ }^{1-2-3}$ The clinical cluster of hypertension, cardiovascular disease, hyperlipemia, hyperuricemia and type 2 Diabetes had been recognized as syndrome $\mathrm{X}$ which is finally named as metabolic syndrome (Met.S) ${ }^{4}$ which was the subjects of this study.

There is a known association between periodontitis and Met.S. The microorganisms associated with periodontitis are diverse. The association between P.gingivalis and periodontitis is well established but the association between P.gingivalis and Met.S is not so evident. Studies have also reported presence of P.gingivalis in periodontitis, but microbiological status in Met.S patients is not yet evaluated. Hence, with this aim in mind, we conducted the present study to evaluate the incidence of P.gingivalis in periodontitis patients with Met.S \& patients with periodontitis without Met.S.

\section{Materials AND Method}

The present study was carried out in the Department of Oral Pathology and Microbiology of Sharad Pawar Dental College. The patients were selected from the Outpatient Department(OPD) of the department of Periodontics, Sharad Pawar Dental College and department of General Medicine, Achaya Vinoba Bhave Rural Hospital Sawangi, Meghe Wardha. Before the start of the study, an informed consent was obtained from all the study subjects.The study was 
Evaluation of Porphyromonas Gingivalis in Periodontitis Patients of Met.S and Periodontitis Patients without Met.S

approved by the Institutional Ethics Committee, Datta Meghe Institute of Medical Sciences, Wardha (Deemed University) Ref. No. DMIMS(DU)/ IEC/ 2013-14/ 127 date-30.09.2013.The study was performed on subjects, which were divided into three groups according to their periodontal status and presence or absence of Met.S.

1. Group I: Periodontally healthy subjects without systemic disorder. $(n=50)$.

2. Group II: Chronic Periodontitis patients without Met.S ( $\mathrm{n}=50)$.

3. Group III: Chronic Periodontitis patients with Met.S $(n=50)$

The Criteria for Periodontal Disease Sites were as follows

1. Probing pocket depth(PPD) $>3 \mathrm{~mm}$, with the help of Williams graduated periodontal probe.

2. Presence of bleeding on probing(BOP).

3. Presence of clinical attachment loss(CAL).

4. Gingival Index score (GI $\geq 2)$

The Criteria for Diagnosis of Met.S were as follows

1. Body Mass Index (BMI), Obesity $>30$ (mass $^{\mathrm{kg}}$ / height $\mathrm{m}^{2}$ )

2. Pressure $(\mathrm{BP})>140 / 90 \mathrm{mmHg}$

3. Triglycerides (TG) $>160 \mathrm{mg} \%$

4. Fasting Blood Sugar(FBS) $>125 \mathrm{mg} / \mathrm{dl}$

5. High -Density Lipoprotein (HDL) $>75 \mathrm{mg} \%$

Detailed clinical history was recorded and clinical assessment was carried out in all the three groups. Subgingival plaque samples were collected and subjected to conventional microbial culture method.

For Group I- Subgingival plaque sample was collected from molar area of periodontally healthy individuals. The sampling site was isolated using cotton rolls and supragingival plaque was removed with the help of sterile cotton. The subgingival plaque sample was then collected using sterile Gracey curettes.

For Group II and III- The sampling sites were isolated with cotton roles and subgingival plaque was removed using sterile cotton. Subgingival plaque samples were obtained using sterile gracy curettes from deepest periodontal pocket. Plaque was subsequently transferred into a sterile container (Himedia) with $0.85 \%$ of sterile saline that was immediately processed. Serial Dilution was done. A serial dilution is the stepwise dilution of a substance in solution. Usually the dilution factor at each step is constant, resulting in a geometric progression of the concentration in a logarithmic fashion. In $100 \mathrm{ml}$ distilled water, $0.85 \mathrm{gms}$ Sodium chloride ( $\mathrm{NaCl}$ - SD fine company) was added. The prepared $9 \mathrm{ml}$ of $0.85 \% \mathrm{NaCl}$ was dispensed into 3 test-tubes (Borosil) each and autoclaved. After autoclaving 5-fold serial dilutions of the sample was made in the test-tube containing $0.85 \% \mathrm{NaCl}$. The serial dilution of samples were done immediately and cultured on agar plates. Brucella Agar Plate with Hemin and Vitamin K1. Hi MP1039 is recommended for the isolation of for P.gingivalis. The prepared culture plate were used for inoculation. The plaque sample which was already serially diluted was added to each medium using streak culture method. Streak culture method is a routinely employed method for bacterial isolation in pure culture. A platinum or nichrome wire loop of 2-4 $\mathrm{nm}$ in internal diameter is used. A loopful of specimen is smeared onto the surface of dried plate near the peripheral area. This is known as primary inoculum. From the primary inoculum, it is spread thinly over the plate by streaking with loop in parallel lines. The streak plate technique is essentially a method to dilute the number of organisms, decreasing the density. This allows for individual colonies to be isolated from other colonies. Each colony is considered "pure," since theoretically, the colony began with an individual cell.Each inoculated petri-plate was sealed with Parafilm (Himedia) to prevent any contamination while incubation, and then placed in an anaerobic jar (Himedia) with AnaeroGas Pack system (Himedia) and incubated at $37^{\circ} \mathrm{c}$ for $48-72$ hours. All the plates are then placed in the anaerogas jar and anaerogas pack is placed inside the jar to maintain the anaerobic condition. The anaerobic gas jar is then placed inside the incubator at $37^{\circ} \mathrm{C}$ for 72 hours.After 72 hours the colonies cultured on Petri plates are subjected to biochemical identification test for confirmation of bacteria intended to be cultured.The identification of a bacterial species is based on factors, including colony morphology, chemical composition of cell walls, biochemical activities, and nutritional requirements. 3 points should be considering during microorganism identification. (Table 1) P.gingivalis is gram negative and Indole Test was positive. 
Evaluation of Porphyromonas Gingivalis in Periodontitis Patients of Met.S and Periodontitis Patients without Met.S

Table 1. Colony Morphology (from agar plates) P. gingivalis

\begin{tabular}{|l|l|l|l|l|}
\hline Shape & Elevation & Edge & Color & Surface \\
\hline Small Rod shaped & Not raised & Irregular & Colourless & Convex \\
\hline
\end{tabular}

\section{OBSERVATIONS AND RESULTS}

In this study, a quantitative analysis of P.gingivalis in periodontitis patients with Met.S and periodontitis patients without Met.S done. The results of the present study were subjected to statistical analysis. The comparison of the P.gingivalis counts in periodontitis patients with Met.S (Group III), periodontitis patients Table 2. Presence of P.gingivalis colonies group wise

\begin{tabular}{|l|l|l|l|l|l|}
\hline Groups & Present & Absent & Total & $\kappa 2$-value \pm SD & p-value \\
\hline Group I & $02(4 \%)$ & $48(96 \%)$ & $50(100 \%)$ & & \\
\hline Group II & $31(62 \%)$ & $19(38 \%)$ & $50(100 \%)$ & $7.55 \pm 1.69$ & \\
\hline Group III & $40(80 \%)$ & $10(20 \%)$ & $50(100 \%)$ & $13.68 \pm 2.48$ & \\
\hline Total & 73 & 77 & 150 & & $\mathrm{p}<0.01$ \\
\hline
\end{tabular}

In Normal controls (Group I), mean total count for P.gingivalis was $0.04 \pm 0.19 \times 10^{5}$ with range of $0.00-1.00$. In Periodontitis patients without Met.S (Group II), mean total count for P.gingivalis was $4.68 \pm 3.99 \times 10^{5}$ with range of $0.00-11.00$. In Periodontitis patients with Met.S (Group III), mean total count for P.gingivalis was $10.94 \pm 0.19 \times 10^{5}$ with range $0.00-20.00$. Thus, the sample suggested that P.gingivalis count in periodontitis patients with Met.S was six times more than periodontitis patients without Met.S. In periodontitis patients without Met.S the Comparison of Total Count for P.Gingivalis in Three Groups (All the Values in $10^{5}$ )

Table 3. Descriptive Statistics of P.gingivalis count in all three groups (All the values in $10^{5}$ )

\begin{tabular}{|l|l|l|l|l|l|l|}
\hline Groups & $\mathbf{N}$ & Mean & Std. Deviation & Std. Error & Minimum & Maximum \\
\hline Normal & 50 & 0.40 & 0.19 & 0.027 & 0.00 & 1.00 \\
\hline $\begin{array}{l}\text { Periodontitis without Met.S } \\
\text { (Group II) }\end{array}$ & 50 & 4.68 & 3.93 & 0.55 & 0.00 & 11.00 \\
\hline $\begin{array}{l}\text { Periodontitis with Met.S } \\
\text { (Group III) }\end{array}$ & 50 & 10.94 & 5.95 & 0.84 & 0.00 & 20.00 \\
\hline
\end{tabular}

Table 4. One way ANOVA for P.gingivalis count in all three groups (All the values in 105)

\begin{tabular}{|l|l|l|l|l|c|}
\hline Source of variation & $\begin{array}{l}\text { Sum of } \\
\text { Squares }\end{array}$ & Df & Mean Square & F & p-value \\
\cline { 1 - 4 } Between Groups & 2992.12 & 2 & 1496.06 & \multirow{2}{*}{88.123} & 0.000 \\
\cline { 1 - 3 } Within Groups & 2495.620 & 147 & 16.97 & & S,p $<0.05$ \\
\cline { 1 - 3 } Total & 5487.74 & 149 & & \\
\hline
\end{tabular}

Archives of Dentistry and Oral Health V1 . I1 . 2018 

without Met.S

The statistical significant difference in total count of P.gingivalis was noted between all groups $(\mathrm{p}=0.000)$. Thus, statistical analysis revealed that Pgingivalis

count was highest in periodontitis patients with Met.S (Group III) than periodontitis patients without Met.S (Group II) and Normal control (Group I). (Table 5)

Table 5. Multiple Comparisons: Tukey Test for P.gingivalis count in all three groups (All the values in 105)

\begin{tabular}{|c|c|c|c|c|c|}
\hline \multirow{2}{*}{\multicolumn{2}{|c|}{ Groups }} & \multirow{2}{*}{$\begin{array}{l}\text { Mean } \\
\text { Difference (I-J) }\end{array}$} & \multirow{2}{*}{ p-value } & \multicolumn{2}{|c|}{ 95\% Confidence Interval } \\
\hline & & & & $\begin{array}{l}\text { Lower } \\
\text { Bound }\end{array}$ & Upper Bound \\
\hline \multirow[t]{2}{*}{ Normal (GroupI) } & $\begin{array}{l}\text { Periodontitis } \\
\text { without Met.S } \\
\text { (GroupII) }\end{array}$ & -4.64 & $0.000 *, p<0.05$ & -6.59 & -2.68 \\
\hline & $\begin{array}{l}\text { Periodontitis } \\
\text { with Met.S(GroupIII) }\end{array}$ & -10.90 & $0.000 *, \mathrm{p}<0.05$ & -12.85 & -8.94 \\
\hline $\begin{array}{l}\text { Periodontitis } \\
\text { patients with } \\
\text { Met.S } \\
\text { (GroupIII) }\end{array}$ & $\begin{array}{l}\text { Periodontitis without } \\
\text { Met.S } \\
\text { (GroupII) }\end{array}$ & 6.26 & $\begin{array}{l}0.000 \\
*, p<0.05\end{array}$ & 4.30 & 8.2 \\
\hline
\end{tabular}

\section{Discussion}

Oral cavity is said to be an open system which is exposed to the environment. So, the possibilities of foreign material entering the system are heightened because of constant intake of food and liquids through the mouth. Large numbers of bacteria in oral cavity can start tissue destruction by activating on host defense cells indirectly, which in turn, produce as well as release mediators which will stimulate the effectors of connective tissue breakdown..$^{5-6-7}$

Met.S refers to a constellation of disturbances including glucose intolerance, central obesity, dyslipidemia (hypertriglyceridemia, elevated nonesterifiedfatty acids (NEFAs), and decreased high-density lipoprotein (HDL) cholesterol, and hypertension. ${ }^{4}$

The microbiological analysis revealed that the mean count for P.gingivalis in periodontitis patients was six times more than control. The results of our study were in an agreement with the study of Ann L. Griffenetal, which showed that P.gingivalis were present in low levels in healthy subjects and increased 11.2-fold in individuals with periodontitis. ${ }^{8-9-10}$

The reasons for increased colonies of P.gingivalis in periodontitis patients could be that, P. gingivalis is best characterized as an opportunistic oral pathogen that inhibits the oral biofilm. Many virulence mechanisms have been identified for P.gingivalis. P.gingivalis has a carbohydrate capsule on its outer surface which prevents opsonization by complement and inhibits phagocytosis and killing by neutrophils. The lipopolysaccharide which is produced by it is not very strong, but it could inhibit chemotaxis and killing by leucocytes. This organism possesses several putative virulence factors (including proteases which degrade immunoglobulin, complement, collagen fibres, hyaluronic acid; adhesins, endotoxins, and cytotoxins) that can directly affect the periodontium or elicit host functions that result in the periodontal disease. ${ }^{11-12-13-14-15-16-17-18}$

The results of our study revealed that mean count for P.gingivalis in periodontitis patients with Met.S was four times more than periodontitis patients without Met.S and 8 times more than controls. Our results are in accordance with the study results by Takahashi etal, 2001 who states that levels of P.gingivalis was increased in periodontitis patients with Met.S as compared to normal patients. ${ }^{19-20}$

The reasons for increased in P.gingivalis levels in periodontitis patients with Met.S could be explained as, P.gingivalis, have the known ability to invade deep vascular endothelium of the periodontium of Met.S patients comprising of T2DM. Once periodontal pathogens were entered and established in the 
Evaluation of Porphyromonas Gingivalis in Periodontitis Patients of Met.S and Periodontitis Patients without Met.S

diabetic host, periodontal infection may aggravate microvascular complications. In periodontitis patients, chronic low-level systemic exposure to periodontal microorganisms exists, that will lead to significant changes in plasma levels of cytokines and hormones. Due to the dynamic nature of the inflamed periodontium, the tissue may act as an endocrinelike source of inflammatory mediators. Among the inflammatory biomarkers, TNF-alpha, CRP, IL$1 \beta$, IL- 6 and IL- 8 and IL- 6 are liberated. Excessive concentrations of TNF-alpha negatively regulate insulin signaling and glucose uptake. Additionally, the elevated levels of soluble TNF receptor 1 and 2 (sTNFalphaRI and sTNF-alphaRII) shown in obese patients may lead to a hyperinflammatory state, thereby increasing the risk for periodontal disease. The hyperinflammatory state may be caused by adipocytes, which secrete proinflammatory cytokines, and providing the link between the pathogenesis of type 2 diabetes, obesity, and periodontal disease. The highest levels of TNF-alpha and sTNFalpha receptors were found in those individuals in the highest quartile for BMI. These observations may suggest, an increased acute phase response is associated with insulin resistance contributing to periodontitis. ${ }^{5-21-22-23}$

One of the complications of diabetes is elevation of blood glucose concentrations (hyperglycemia). Hyperglycemia leads to the formation of AGEs. These AGEs make endothelial cells and monocytes more susceptible to stimuli that will then induce the cells to produce inflammatory mediators. Some author suggested that AGE accumulations in the gingival tissue will leads to increased vascular permeability, and increased breakdown of collagen fibers, and more destruction of both nonmineralized connective tissue and mineralized bone. As diabetes is a metabolic disorder and periodontal disease is an infectious disease, the pathophysiological relationship between these two entities is, through the ability of both conditions to induce an inflammatory response, because of AGE or bacterial accumulation, respectively, which further leads to the production of inflammatory mediators. ${ }^{5-24-25-26-27}$

The following conditions in Met.S favor the growth of P.gingivalis-

P.gingivalis attached to the components of extracellular matrix and to the Type I collagen. This helps in colonization of P.gingivalis to gingival margin. The arg-gingipain protease of P.gingivalis helps either directly or indirectly in this attachment. Directly by acting as an adhesion and indirectly by affecting the expression of an adhesion. A strong association between gingival protease activity and periodontal infection were found in studies. In Met.S serum levels of Cystatin C (cysteine protease) are synthesized at constant rate. ${ }^{28}$ Therefore it could be hypothesized that constant increased in protease in Met.S patients favours the colonization of P.gingivalis. Other reasons for increase colonization of P.gingivalis could be that P.gingivalis has the ability to identify receptors on gingival epithelial cell receptors even in low chronic inflammatory condition, P.gingivalis also has the property to respond and adopt to oxidative stress as they are oxidative stress resistance. P.gigivalis growth requirements include iron, which is provided by elevated iron stores in GCF of Met.S patients. ${ }^{29}$

Thus, Patients diagnosed with Met.S are thought to be at higher risk due to a compromised immune system. Infectious and opportunistic microbes responsible for periodontal infection may thus bring a burden onto the rest of the body and release products that elicit an inflammatory response.

\section{ConClusion}

The fact that increases in colonization of periodontal pathogens may be attributed to the assumption that, Periodontitis releases proinflammatory cytokines and ROS at the site of inflammation, leading to oxidative stress situation..$^{30}$ This will contribute to aggravate existing Met.S.

\section{REFERENCES}

[1] Joy Kumar Chakma, Sanjay Gupta. Review Article. Lifestyle and Non-Communicable Diseases: A double edged sword for future India. Indian Journal Of Community Health, Oct - Dec 2014, Vol 26, Issue no 04.

[2] National institute of medical sciences, Indian Council of Medical Research ICMR(2009), IDSP Non communicable disease Risk factor survey, Phase I state of India, 2007-2008. National Institute of medical statistics and division of non communicable disease, Indian council of Medical research, New Delhi, India. 
Evaluation of Porphyromonas Gingivalis in Periodontitis Patients of Met.S and Periodontitis Patients without Met.S

[3] Divakaran B, Muttapillymyali J, Shalini K. Lifestyle riskfactors of noncommunicable diseases: awareness among school children. Indian J Cancer. 2010 Jui47 Suppl 1:9-13.

[4] Aye: Metabolic syndrome, J Metabolic syndrome 2014:3;4.

[5] Jemin Kim and Boston. Periodontal disease and systemic conditions: a bidirectional Relationship. NIHOdontology. September 2006; 94(1): 10-21.

[6] Sorsa T, Ingman T, Suomalainen K, Haapasalo M, Konttinen YT, Lindy O, Saari H, Uitto VJ. Identification of proteases from periodontopathogenic bacteria as activators of latent human neutrophil and fibroblast-type interstitial collagenases. Infect Immun. 1992;60:4491-5

[7] Loesche WJ, Gusberti F, Mettraux G, Higgins T, Syed S. Relationship between oxygen tension and subgingival bacterial flora in untreated human periodontal pockets. Infect Immun. 1983;42:659-67.

[8] Ann L Griffen, Clifford J Beall, James H Campbell, Noah D Firestone, Purnima S Kumar, Zamin K Yang, Mircea Podar, and Eugene J Leys Distinct and complex bacterial profiles in human periodontitis and health revealed by $16 \mathrm{~S}$ pyrosequencing ISME J. 2012 Jun; 6(6): 1176-1185.

[9] GrifenAL,BeallCJ,FirestoneND,GrossEL, DiFranco JM, Hardman JH, et al. CORE: a phylogeneticallycurated 16S rDNA database of the core oral microbiome. PLoS ONE. 2011;6:e19051.

[10] Kumar PS, Griffen AL, Barton JA, Paster BJ, Moeschberger ML, Leys EJ. New bacterial species associated with chronic periodontitis. J Dent Res. 2003;82:338-344.

[11] Ljiljana Kesic1,Jelena Milasin2, Marija Igic3, Radmila Obradovic. Microbial Etiology Of Periodontal Disease - Mini Review. FACTA Universitatis Series: Medicine and Biology 2008,Vol.15, No 1 pp. 1 - 6.

[12] Slots J, Genco RJ. Black-pigments Bacteroides species, Capnocytophaga species, and Actinobacillus actinomycetemcomitans in human periodontal disease:Virulence factors in colonization, survival, and tissue destruction. J Dent Res 1984; 63: 412-421.
[13] Lantz MS. New insights into mechanisms of bacterial pathogenesis in periodontitis. Curr Opin Periodontol 1996; 3: 10-18.

[14] Isogai H, Isogai E, Yoshimura F, Suzuki T, Kagota W, Takano K. Specific inhibition of adherence of an oral strain of Bacteroides gingivalis 381 to epithelial cells by monoclonal antibodies against the bacterial fimbriae. Arch Oral Biol 1988; 37: 479- 485.

[15] Papaioannou W, van Steenberghe D, Cassiman J-J, van Eldere J, Quirynen M. Comparison of fluorescence microscopy and culture assays to quantitative adhesion of Porphyromoina sgingivalis to mono- and multi-layered pocket epithelium cultures. J Periodontol 1999; 70: 618-625.

[16] Watanabe K, Yamaji Y, Umemoto T. Correlation between celladherent activity and surface structure in Porphyromonas gingivalis. Oral Microbiol Immunol 1992; 7: 357-363.

[17] Sandros J, Papapanou PN, Nannmark U, Dahlen G. Porphyromonas gingivalis invades human pocket epithelium in vitro. J Periodont Res 1994; 29: 62-69.

[18] Du L, Pellen-Mussi P, Chandad F, Mouton C, Bonnaure-Mallet M. Fimbriae and the hemagglutinating adhesin HA-Ag2 mediate adhesion of Porphyromonas gingivalis to epithelial cells. Infect Immunol 1997; 65: 38753881

[19] Yokota T, Oritani K, Takahashi I, et al. Adiponectin, a new member of the family of soluble defense collagens, negatively regulates the growth of myelomonocytic progenitors and the functions of macrophages. Blood. 2000;96:1723-32.

[20] Yusuke Takahashi, Hisashi Yoshimoto, Daisuke Kato, Nobushiro Hamada, Munetaka Arai, Toshio Umemoto. Reduced fimbria-associated activities ofPorphyromonas gingivalis induced by recombinant fimbrial expression FEMS Microbiology Letters 195 (2001) 217-222.

[21] Pradhan AD, Ridker PM. Do atherosclerosis and type 2 diabetes share a common inflammatory basis? Eur Heart J. 2002;23:831-4. 
Evaluation of Porphyromonas Gingivalis in Periodontitis Patients of Met.S and Periodontitis Patients without Met.S

[22] Yudkin JS, Stehouwer CD, Emeis JJ, Coppack SW. C-reactiveproteininhealthysubjects:associations with obesity, insulin resistance, and endothelial dysfunction: a potential role for cytokines originating from adipose tissue? Arterioscler Thromb Vasc Biol. 1999;19:972-8.

[23] Bluher M, Fasshauer M, Tonjes A, Kratzsch J, Schon MR, Paschke R. Association of interleukin-6, C-reactiveprotein,interleukin-10andadiponectin plasma concentrations with measures of obesity, insulin sensitivity and glucose metabolism. Exp Clin Endocrinol Diabetes. 2005;113:534-7.

[24] Christgau M, Palitzsch KD, Schmalz G, Kreiner $\mathrm{U}$, Frenzel S. Healing response to nonsurgical periodontal therapy in patients with diabetes mellitus: clinical, microbiological, and immunologic results. J Clin Periodontol. 1998;25:112-24.

[25] Grossi SG, Zambon JJ, Ho AW, Koch G, Dunford RG, Machtel EE, et al. Assessment of risk for periodontal disease. I. Risk indicators for attachment loss. J Periodontol. 1994;65:260-7.
[26] Stewart JE, Wager KA, Friedlander AH, Zadeh HH. The effect of periodontal treatment on glycemic control in patients with type 2 diabetes mellitus. J Clin Periodontol. 2001;28:306-10

[27] OffenbacherS, SalviGE.Induction ofprostaglandin release from macrophages by bacterial endotoxin. Clin Infect Dis. 1999;28:505-13.

[28] M Tokuda, M Duncan, M I Cho, H K Kuramitsu. Role of Porphyromonas gingivalis protease activity in colonization of oral surface. Infec Immun. oct 1996:64(10);4067-4073.

[29] Enrico Marchetti, Annalisa Monaco, Laura Procaccini, Stefano Mummolo, Roberto Gatto, Stefano Tetè, Alberto Baldini, Simona Teccol and Giuseppe Marzo. Review article- Periodontal disease: the influence of metabolic syndrome. J. Nutrition \& Metabolism 2012, 9:88.

[30] Sergio Granados, Nuri-El-Azem, Jose L. Quiles, Patrica Perez-Lopez, Adrian Gonzalez, and MCarmen Ramirez- Tortosa. Relationsip between cardiovascular risk factor and periodontal disease: Current Knowledge.193-216.

Citation: Dr. Ashwini Walke, Dr. Tushar Bhagat, Dr. Abdulmajeed Mutairan Alanazi. Evaluation of Porphyromonas gingivalis in periodontitis patients of Met.S and periodontitis patients without Met.S. Archives of Dentistry and Oral Health. 2018; 1(1): 07-13.

Copyright: (C) 2018 Dr. Ashwini Walke, Dr. Tushar Bhagat, Dr. Abdulmajeed Mutairan Alanazi. This is an open access article distributed under the Creative Commons Attribution License, which permits unrestricted use, distribution, and reproduction in any medium, provided the original work is properly cited. 